\title{
Design of GRIN laser beam shaping system
}

\author{
David L. Shealy ${ }^{a}$ and Shao-Hua Chao ${ }^{b}$ \\ ${ }^{a}$ University of Alabama at Birmingham, Department of Physics, 1530 3rd Ave. S, CH310, \\ Birmingham, AL 35294-1170, USA; \\ ${ }^{b}$ Hualien Teacher College,Room 202, Sciences Building, 123 Hua-hsi, Hua Lien, Taiwan 970
}

\begin{abstract}
Geometrical optics is used for design of gradient-index (GRIN) laser beam shapers with the conditions of conservation of energy and constant optical path length for all rays passing through the system. The exact ray intercepts for a Gaussian to top-hat beam transform at the output plane are the ray trace target values used during the optimization process. After constructing a beam shaping merit function, the commercial software ZEMAX has been used to minimize the merit function for a well known two-element plano-aspheric beam shaper to establish the effectiveness of this new beam shaping merit function. Then, this method is used to design of several GRIN laser beam shapers while using ZEMAX's catalog GRADIUM elements from LightPath glass types. The optical component shape and spacing parameters are also used for optimization variables. Both spherical surfaces and conic surfaces of the different elements of the GRIN laser beam shaper are studied. The ZEMAX software was used for performance analysis of the GRIN beam shapers and is discussed.
\end{abstract}

Keywords: Laser beam shaping, geometrical optics, optimization, GRIN, irradiance mapping

\section{INTRODUCTION}

There are many applications of lasers in technology, lithography, and optical devices which benefit from a uniform beam profile. It is important to study ways to convert a input Gaussian beam into a more uniform output beam. There are reflective,${ }^{1-4}$ refractive,${ }^{4-11}$ and diffractive ${ }^{12-16}$ methods to shaping laser beam profiles. Geometrical optics has been applied to the design of the laser beam shaping systems. The techniques have included the differential equations method and the genetic algorithm (GA) optimization method. ${ }^{11,17-21}$ In the optimization method, there is no need to solve the differential equations for the optical element surfaces contours of the beam shapers. Rather, the optimization programs locate system parameters, which characterize the beam shaper and which minimizes the beam shaping merit function that incorporates the conditions of conservation of energy and constant optical path length. The genetic algorithm (GA) has been applied to solve the optimization problems when both discrete and continuous parameters are required to define the system. Evans and Shealy ${ }^{11,17}$ have proposed serveral beam shaping merit functions and have used the GA to study two- and three-element GRIN beam shapers. The global optimization method of the commercial software ZEMAX ${ }^{22}$ optical design and analysis program includes the GA method along with other methods. Therefore, it is desirable to apply the optimization programs of ZEMAX for design of laser beam shapers.

In this paper, we present a new way to incorporate the conditions of conservation of energy and constant optical path length in the definition of a beam shaping merit function that has been used with ZEMAX ${ }^{22}$ optimization methods for design of GRIN beam shapers. In Sect. 2, we derive an expression for the output ray coordinate as a function of the input ray coordinate for a plane wave Gaussian beam transformed into a plane wave top-hat output beam. Then, we define a beam shaping merit function and give a brief describation on the ZEMAX optimization methods that will be used to design laser beam shapers by minimization of the merit function. In Sect. 3, results of using this method to design laser beam shapers are presented and discussed. The ZEMAX software has been applied to analyze the designed systems. Conclusions are given in Sect. 4.

Further author information: (Send correspondence to D.L.S.)

D.L.S.: E-mail: dls@uab.edu, Telephone: 12059348068

S.H.C.: E-mail: chao@sparc2.nhltc.edu.tw, FAX: 011-886-38-224-972

Copyright 2004 Society of Photo-Optical Instrumentation Engineers.

This paper will be published in Proc. SPIE 5525, Sept. 2006, and is made available as an electronic preprint with permission of SPIE. One print or electronic copy may be made for personal use only. Systematic or multiple reproduction, distribution to multiple locations via electronic or other means, duplication of any material in the paper for a fee or for commercial purposes, or modification of the content of the paper are prohibited. 


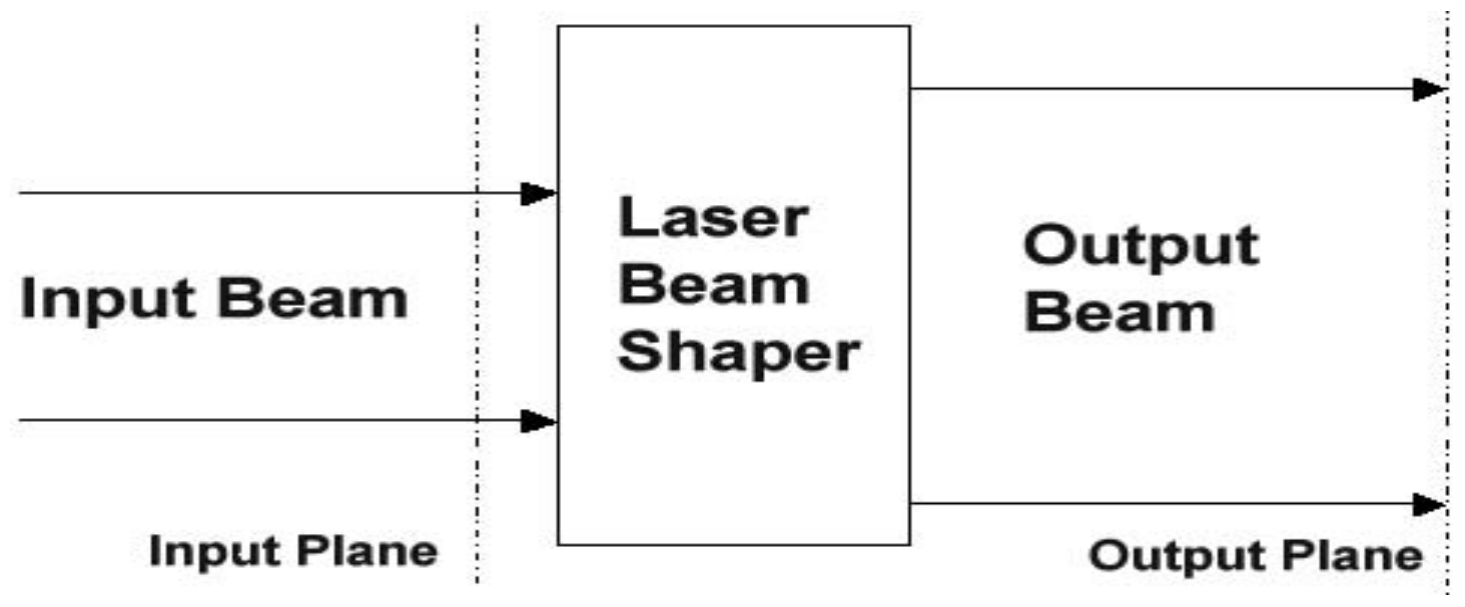

Figure 1. Symbolic diagram of a laser beam shaping system.

\section{DESIGN METHOD}

This section describes a method for design of laser beam shapers by minimizing a beam shaping merit function. First, the conservation of energy condition is used to evaluate a relationship between the input and output ray coordinates. Then, a beam shaping merit function is defined. This merit function includes the conditions of conservation of energy and constant optical path for all rays passing through the system. Finally, the optimization methods of ZEMAX are briefly discussed.

\subsection{Application of Conservation of Energy}

For a lossless laser beam shaping system, the total energy of the input beam is mapped into the output beam. Figure 1 illustrates the components of a laser beam shaping system. In a rotationally symmetric optical system, a group of rays with irradiance $I_{i n}(r)$ passing through the optical system has the following total energy at the input plane

$$
E_{\text {in }}=\int I_{i n}(r) d a
$$

where $d a$ is the differential area on the input plane. The total energy over the output plane is given by

$$
E_{\text {out }}=\int I_{\text {out }}(R) d A,
$$

where $I_{\text {out }}(R)$ and $d A$ are the irradiance and differential area at the output plane, respectively.

To satisfy conservation of energy for the beam transform performed by the laser beam shaper, the input energy $E_{\text {in }}$ must be equal to the output energy $E_{\text {out }}$. For an input Gaussion beam, the beam profile is given by

$$
I_{\text {in }}(r)=\exp \left[-2\left(\frac{r}{r_{0}}\right)^{2}\right]
$$

where $r$ is the radial distance from the optical axis, and $2 r_{0}$ is the diameter of the Gaussian beam waist. For a lossless transformation of a Gaussian beam into an uniform irradiance output beam, the conservation of energy condition requires

$$
2 \pi \int_{0}^{r_{\max }} \exp \left[-2\left(\frac{r}{r_{0}}\right)^{2}\right] r d r=2 \pi \int_{0}^{R \max } C R d R,
$$

Copyright 2004 Society of Photo-Optical Instrumentation Engineers.

This paper will be published in Proc. SPIE 5525, Sept. 2006, and is made available as an electronic preprint with permission of SPIE. One print or electronic copy may be made for personal use only. Systematic or multiple reproduction, distribution to multiple locations via electronic or other means, duplication of any material in the paper for a fee or for commercial purposes, or modification of the content of the paper are prohibited. 
where $r$ and $R$ are the radial distances at the input and output planes, $r_{\max }$ and $R_{\max }$ are the corresponding maximum ray coordinates, and $C$ is the constant output irradiance. Evaluating the integrals and solving for $C$ leads to

$$
C=-\frac{1}{2}\left(\frac{r_{0}}{R_{\max }}\right)^{2}\left\{\exp \left[-2\left(\frac{r_{\max }}{r_{0}}\right)-1\right]\right\} .
$$

Then, to find the relationship between the output ray coordinate $R$ and the input ray coordinate $r$, apply the conservation of energy condition with variable upper limits

$$
\int_{0}^{r} \exp \left[-2\left(\frac{r}{r_{0}}\right)^{2}\right] r d r=\int_{0}^{R} C R d R .
$$

Integrating and solving for $R$ gives

$$
R(r)=\left[\frac{1-\exp \left[-2\left(\frac{r}{r_{0}}\right)^{2}\right]}{1-\exp \left[-2\left(\frac{r_{\max }}{r_{0}}\right)^{2}\right]}\right]^{\frac{1}{2}} R_{\max } .
$$

This gives the theoretical ray height $R(r)$ on the output plane for a specific incident ray of radius $r$ at the input plane for which energy is conserved by the beam transform. The expression for $R(r)$ will be useful when defining the beam shaping merit function in Sect. 2.2.

\subsection{Merit Function}

After applying the conservation of energy condition to obtain the output plane ray intercept for a particular incident ray, the condition for constant optical path implies that for a collimated incident beam along z-axis the output rays will also be collimated along the z-direction. Therefore, the theoretical output plane ray intercept will be equal to the ray intercept on the optical surface just before the output plane. By putting these considerations into the definition of the beam shaping merit function, then the optimization process of laser beam shaping optics will lead to systems which satisfy the beam shaping equations of conservation of energy and constant optical path length condition.

Assume that there are $N$ rays incident upon the beam shaper. Then, the $i^{t h}$ ray will have the radial coordinate $r_{i}$ at the input plane

$$
r_{i}=(i-1)\left[\frac{r_{\max }-r_{\min }}{N-1}\right],
$$

where $r_{\max }$ and $r_{\min }$ are the maximum and minimum of the radial distances on the input plane.

Assume that a beam shaping merit function, $\digamma$, consists of two parts. The first part of the beam shaping merit function is labed by $u_{r}$, which is the weighted sum of the absolute value of the differences between the exact theoretical ray intercept on the output plane and the ray intercept point with the assumed optics configuration under optimization. Then, $u_{r}$ is given by

$$
u_{r}=\sum_{i=1}^{N}\left[\left|\left(r_{i i}-r_{i e}\right)\right|+\left|\left(r_{i 2}-r_{i e}\right)\right|\right],
$$

where $r_{i e}$ is the exact theoretical interception point at the output plane of the incident ray, $r_{i i}$ and $r_{i 2}$ are the ray interception points of the optimized optics configuration at the output plane and the optical surface before the output plane, respectively.

Copyright 2004 Society of Photo-Optical Instrumentation Engineers.

This paper will be published in Proc. SPIE 5525, Sept. 2006, and is made available as an electronic preprint with permission of SPIE. One print or electronic copy may be made for personal use only. Systematic or multiple reproduction, distribution to multiple locations via electronic or other means, duplication of any material in the paper for a fee or for commercial purposes, or modification of the content of the paper are prohibited. 
The second part of the beam shaping merit function is labeled by $u_{\theta}$ for the collimation of the output rays, which is given by

$$
u_{\theta}=\sqrt{\sum_{i=1}^{N}\left(1-\gamma_{i}\right)}
$$

where $\gamma_{i}$ is the directional cosine of the $i^{\text {th }}$ incident ray at the output plane in the z-direction, i.e., along the optical axis. Then, the beam shaping merit function $\digamma$ is given by

$$
\digamma=w_{r} u_{r}+w_{\theta} u_{\theta}=w_{r} \sum_{i=1}^{N}\left[\left|\left(r_{i i}-r_{i e}\right)\right|+\left|\left(r_{i 2}-r_{i e}\right)\right|\right]+w_{\theta} \sqrt{\sum_{i=1}^{N}\left(1-\gamma_{i}\right)}
$$

where $w_{r}$ and $w_{\theta}$ are the weighting factors for each part of the beam shaping merit function. This merit function has been minimized during the optimization process for the design of laser beam shapers.

\subsection{Optimization Methods Used in ZEMAX}

The optimization methods in ZEMAX have been applied to the design of several laser beam shaping optical systems. These methods include the global optimization and the hammer optimization. The global optimization applies a combination of genetic algorithms (GA), conventional damped least square method, multistart,and some expert system heuristics to find the new design forms. This usually will find a good design form which is the starting system for the hammer optimization. The hammer optimization searches exhaustively for the optimum system. The optimum system is the system with the smallest merit function. Both methods will usually take a long time to run on desktop computers with typical run times lasting for many hours or several days to yield effective designs. The ZEMAX optimization programs are very useful. Even so, there is no guarantee that a global optimized system will eventually be found for optical configuration under study. For detailed description of the optimization methods in ZEMAX please refer to the ZEMAX manual.

\section{RESULTS}

In order to evaluate the performance of this optimization approach for design of laser beam shapers, it is necessary to first apply this design method to a beam shaper with known solutions to confirm that the new optimization methods leads to acceptable solutions. Then, the optimization methods can be applied to more complex beam shaping configurations where solutions are unknown. First, we seek to minimize the beam shaping merit function $\digamma$ by the ZEMAX ${ }^{22}$ optimization methods for design of a two plano-aspheric lens beam shaper, which transforms a rotationally symmetric Gaussian laser beam into a uniform irradiance output beam. Then, the beam shaping optimization method is used to design multi-element gradient index (GRIN) lenses.

\subsection{Two Plano-aspheric Beam Shaper}

The two plano-aspheric laser beam shaper has been studied extensively by Frieden, ${ }^{5}$ Rhodes and Shealy, ${ }^{6}$ Evans and Shealy, ${ }^{17}$ Hoffnagle and Jefferson, ${ }^{10}$ and others. Some studied the Galilean configuration of the pair of plano-aspheric lenses where the first element has negative power, and others studied the Keplerian configuration where the first element has positive power. Both configurations have been studied by the method described in Sect. 2 and results are presented in this section.

The aspherical lens surface is described by the even aspheric surface equation

$$
z(r)=\frac{c r^{2}}{1+\sqrt{1-(1+k) c^{2} r^{2}}}+\sum_{j=2}^{6} A_{2 j} r^{2 j}
$$

Copyright 2004 Society of Photo-Optical Instrumentation Engineers.

This paper will be published in Proc. SPIE 5525, Sept. 2006, and is made available as an electronic preprint with permission of SPIE. One print or electronic copy may be made for personal use only. Systematic or multiple reproduction, distribution to multiple locations via electronic or other means, duplication of any material in the paper for a fee or for commercial purposes, or modification of the content of the paper are prohibited. 


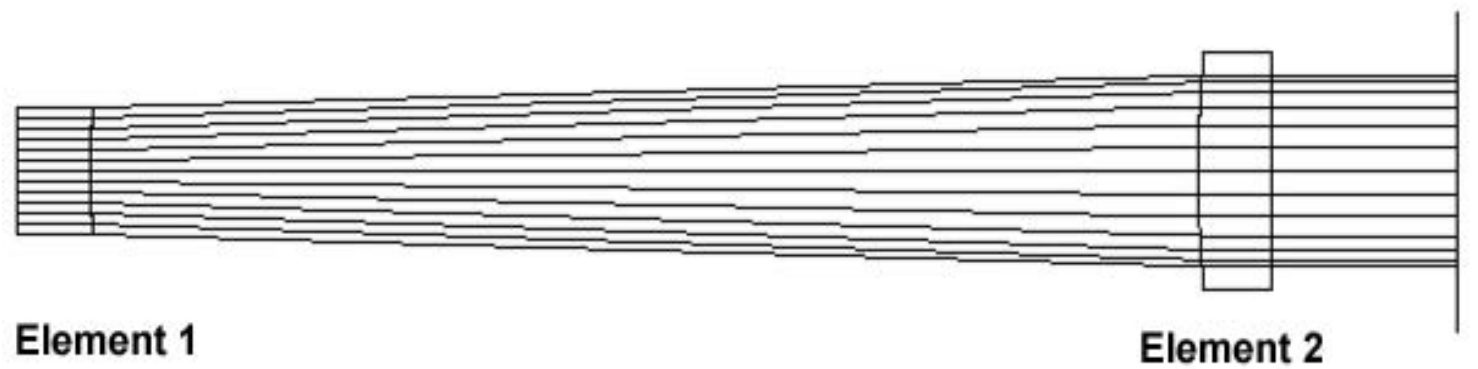

\section{Element 1}

\section{Element 2}

Figure 2. Two-dimensional cross section of optical layout for the designed Galilean configuration of a two-plano-aspheric beam shaper. Element 1 is a plano-concave aspheric lens with negative power, and element 2 is a convex aspheric-plano lens.

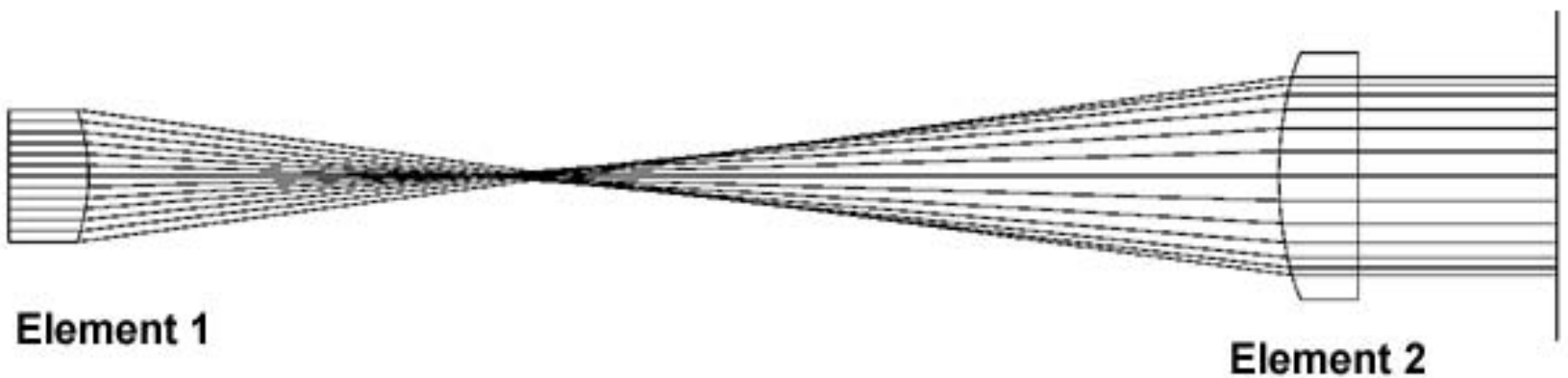

Figure 3. Two-dimensional cross section of optical layout for the designed Keplerian configuration of a two-plano-aspheric beam shaper. Element 1 is a plano-convex aspheric lens with positive power, and element 2 is a convex aspheric-plano lens.

where $\mathrm{z}$ is the sag of the aspherical lens surface, $\mathrm{r}$ is the radial distance from the $\mathrm{z}$-axis, $c$ is the curvature of the aspherical lens surface, $k$ is the conic constant and $A_{2 j}$ are the aspherical deformation coefficients.

The variables for applying ZEMAX ${ }^{22}$ optimization methods are $c, k$, and $A_{2 j}$. The beam shaping system will expand the input Gaussian profile beam of $8 \mathrm{~mm}$ radius to an uniform profile output beam of $12 \mathrm{~mm}$ radius. The lens material is chosen to be $\mathrm{CaF}_{2}$, with wavelength as $589 \mathrm{~nm}$, and the lens units is millimeters. The lens thickness is chosen to be $10 \mathrm{~mm}$ for both lenses, and the separation for two lenses is $150 \mathrm{~mm}$, the distance from the last surface of the $2^{\text {nd }}$ lens to the output plane is $25 \mathrm{~mm}$ for both designs. Figures 2 and 3 shows a cross-section of the 3D layout diagrams for the designed Galilean and Keplerian configurations of a two-lens systems beam shaper, respectively. Both figures show that the input put rays on an equally space grid have been redistributed to form a uniform irradiance of the output beam with good collimation along the optical axis. Typical x-scan and y-scan of relative illumination on the output plane for both type of designs is shown in Fig. 4, which confirms that the input Gaussian beam has been transformed to a more uniform output beam in both $\mathrm{x}$ and $\mathrm{y}$ directions. Therefore, the laser beam shaping method described in Sect. 2 has been successively applied to the design of the two plano-aspheric lenses beam shaper as reported by others. Table 1 shows the system parameters of the designed Galilean and Keplilean type two lens reshaping optical systems using the beam shaping merit function and optimization procedures described in this paper.

Copyright 2004 Society of Photo-Optical Instrumentation Engineers.

This paper will be published in Proc. SPIE 5525, Sept. 2006, and is made available as an electronic preprint with permission of SPIE. One print or electronic copy may be made for personal use only. Systematic or multiple reproduction, distribution to multiple locations via electronic or other means, duplication of any material in the paper for a fee or for commercial purposes, or modification of the content of the paper are prohibited. 


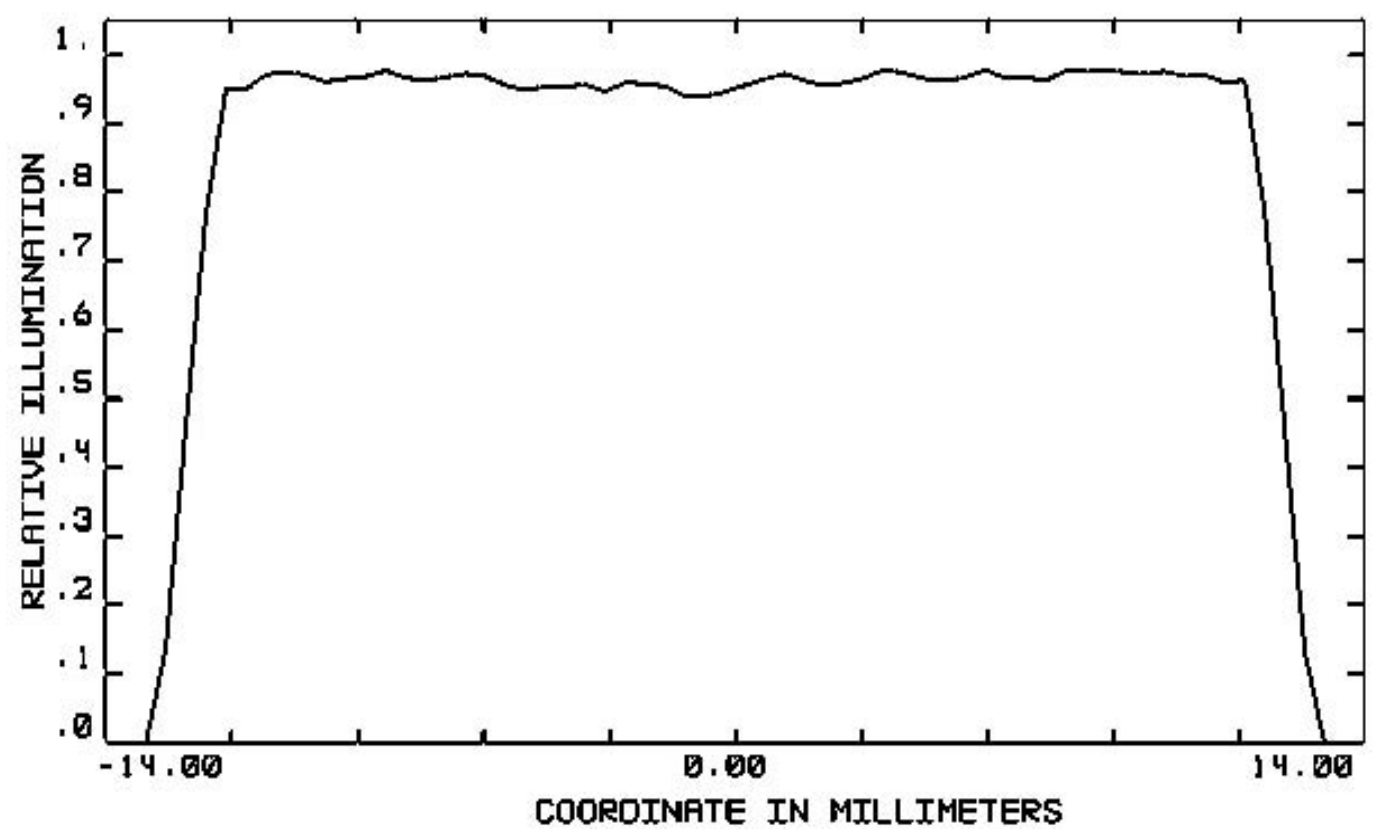

Figure 4. Typical x-scan and y-scan plots of relative irradiance over the output plane of the designed two-lens laser beam shapers.

Table 1. The designs of the Keplerian and Galilean type two-plano aspheric lens laser beam shaper.

\begin{tabular}{|c|c|c|}
\hline & Galilean type design & Keplerian type design \\
\hline First lens & $C a F_{2}$ & $C a F_{2}$ \\
\hline Radius of curvature of the first surface & infinity & infinity \\
Radius of curvature of the second surface & 51.11723 & -19.86988 \\
Conic constant of the second surface & -2.410617 & -5.829718 \\
$\mathrm{~A}_{4}$ of the second surface & $-5.9916339 \mathrm{e}-05$ & $5.3579177 \mathrm{e}-06$ \\
$\mathrm{~A}_{6}$ of the second surface & $8.8355242 \mathrm{e}-09$ & $6.1085278 \mathrm{e}-09$ \\
$\mathrm{~A}_{8}$ of the second surface & $2.6636398 \mathrm{e}-09$ & $2.1785474 \mathrm{e}-10$ \\
$\mathrm{~A}_{10}$ of the second surface & $4.032501 \mathrm{e}-12$ & $-8.8595179 \mathrm{e}-12$ \\
$\mathrm{~A}_{12}$ of the second surface & $-2.1488254 \mathrm{e}-13$ & $4.2142567 \mathrm{e}-14$ \\
\hline Second lens & $C a F_{2}$ & $C a F_{2}$ \\
\hline Radius of curvature of the second surface & infinity & infinity \\
Radius of curvature of the first surface & 119.6847 & 45.89605 \\
Conic constant of the first surface & -4.711986 & 0.4956102 \\
$\mathrm{~A}_{4}$ of the first surface & $2.023397 \mathrm{e}-06$ & $2.6258956 \mathrm{e}-06$ \\
$\mathrm{~A}_{6}$ of the first surface & $-3.8667856 \mathrm{e}-08$ & $-1.118072 \mathrm{e}-08$ \\
$\mathrm{~A}_{8}$ of the first surface & $-1.1722665 \mathrm{e}-10$ & $-1.5138836 \mathrm{e}-10$ \\
$\mathrm{~A}_{10}$ of the first surface & $1.979421 \mathrm{e}-12$ & $1.6422034 \mathrm{e}-12$ \\
$\mathrm{~A}_{12}$ of the first surface & $-6.7451487 \mathrm{e}-15$ & $-3.0263946 \mathrm{e}-15$ \\
\hline Thickness of first lens & 10 & 10 \\
Thickness of second lens & 10 & 10 \\
Separation of 1st and 2nd lens & 150 & 150 \\
Separation of 2nd lens and output plane & 25 & 25 \\
\hline
\end{tabular}

Copyright 2004 Society of Photo-Optical Instrumentation Engineers.

This paper will be published in Proc. SPIE 5525, Sept. 2006, and is made available as an electronic preprint with permission of SPIE. One print or electronic copy may be made for personal use only. Systematic or multiple reproduction, distribution to multiple locations via electronic or other means, duplication of any material in the paper for a fee or for commercial purposes, or modification of the content of the paper are prohibited. 


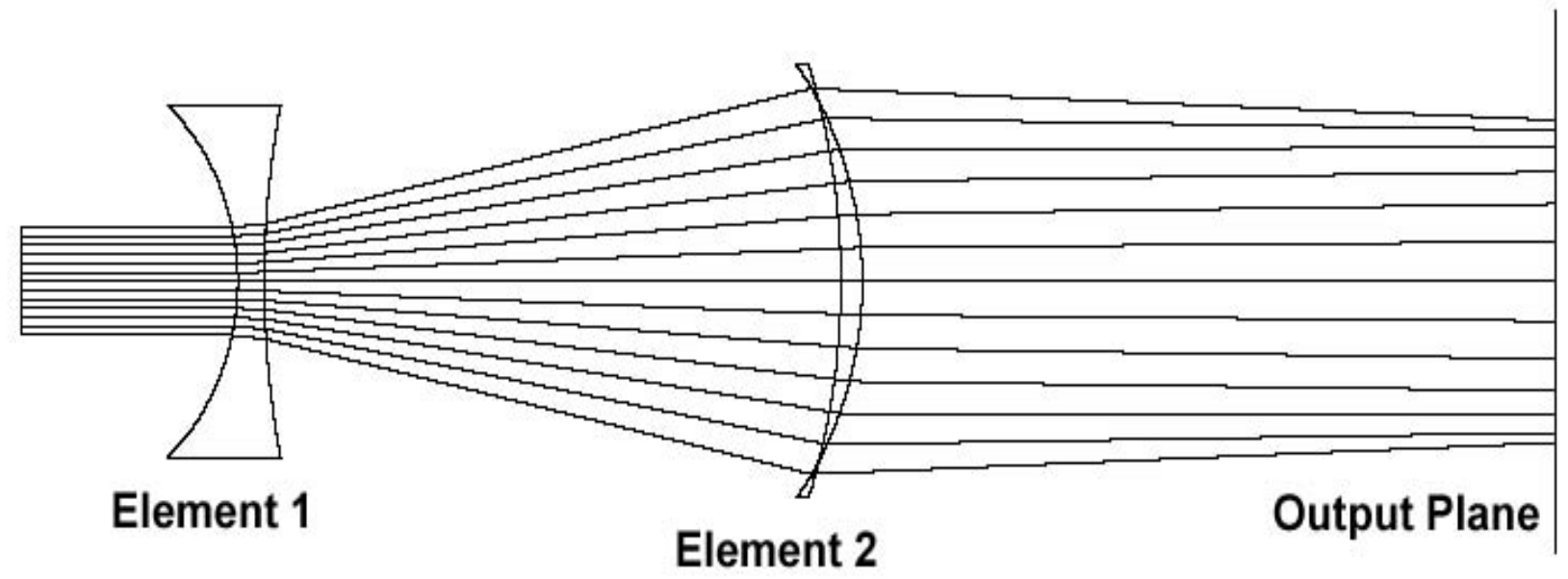

Figure 5. Ray trace of two-spherical GRIN lens beam shaper

\subsection{Multi-element GRIN Laser Beam Shaper}

Now consider design of multi-element GRIN lens beam shaper by using the method described in Sect. 2. Assume that The radius of the input Gaussian beam is $4 \mathrm{~mm}$ and that of the output beam is $12 \mathrm{~mm}$. The type of GRIN lens material is a variable, which is chosen by the ZEMAX optimization program from the GRADIUM GRIN lens of LightPath glass catalog. The other optimization variables are the thickness between surfaces, the radii of curvature of surfaces, the conic constant of the surfaces, and $\Delta z$ which is a variable in the equation of index of refraction of the GRADIUM GRIN lens of LightPath. There are 12 different cataloged GRADIUM GRIN lenses available from LightPath. The ZEMAX optimization program will choose different GRIM materials accordingly along with the other variables while minimizing the merit function $\digamma$. Initially, the conic constant of all the surfaces is set to zero. The spherical GRADIUM GRIN lenses are considered first. Figure 5 illustrates limitations of two spherical GRIN lenses to achieve good beam shaping results. It is clear from Fig. 5 that the optimization process was not able to achieve good collimation of output beam, and from the $\mathrm{x}$ - and $\mathrm{y}$-scans of relative irradiance on the output plane, there is approximately a $10 \%$ variation in uniformity of irradiance over the output plane, or approximately twice as much variation as shown in Fig. 4. The beam shaping performance is improved by allowing a lens surface to include a non-zero conic constant as optimization variables. Figure 6 shows a ray trace of a conic-spherical GRIN lens beam shaper located by this design process. The output beam profile for the lens system shown in Fig. 6 has similar relative irradiance uniformity plots as shown in Fig. 4.

A number of multi-element (2, 3, and 4 elements) laser beam shaping systems have been optimized and analyzed by ZEMAX. These optimization of multi-element GRIN beam shapers requires long computing times, since for each different cataloged GRADIUM GRIN lenses used during an optimization, the corresponding gradient index of refraction must be evaluated as part of the ZEMAX optimization program.

The results obtained for 2 and 3 elements of aspherical cataloged GRADIUM GRIN lenses reshaping optical systems are represented in Figs. 7 and 8. The relative relative illumination plots over the output planes for these beam shapers are similar to Fig. 4. Figures 7 and 8 show that the desired reshaping of Gaussian laser beam profile into the top-hat output beam profile has been obtained. Table 2 shows the system parameters of the designed two and three elements of cataloged GRADIUM GRIN lens reshaping optical systems.

Copyright 2004 Society of Photo-Optical Instrumentation Engineers.

This paper will be published in Proc. SPIE 5525, Sept. 2006, and is made available as an electronic preprint with permission of SPIE. One print or electronic copy may be made for personal use only. Systematic or multiple reproduction, distribution to multiple locations via electronic or other means, duplication of any material in the paper for a fee or for commercial purposes, or modification of the content of the paper are prohibited. 


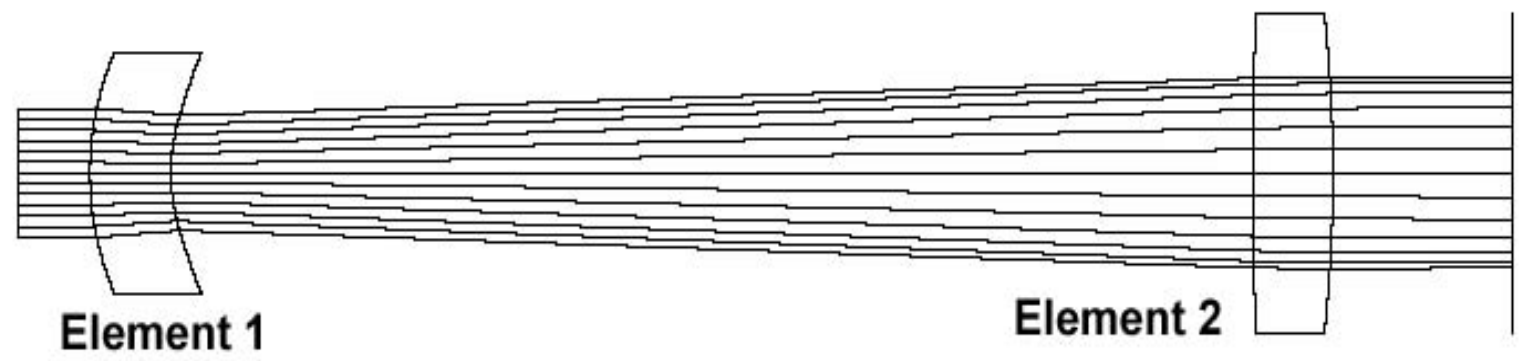

Figure 6. Two-element GRIN beam shaper with one conic surace shape for the second optical surface while the remaining surfaces are spherical.

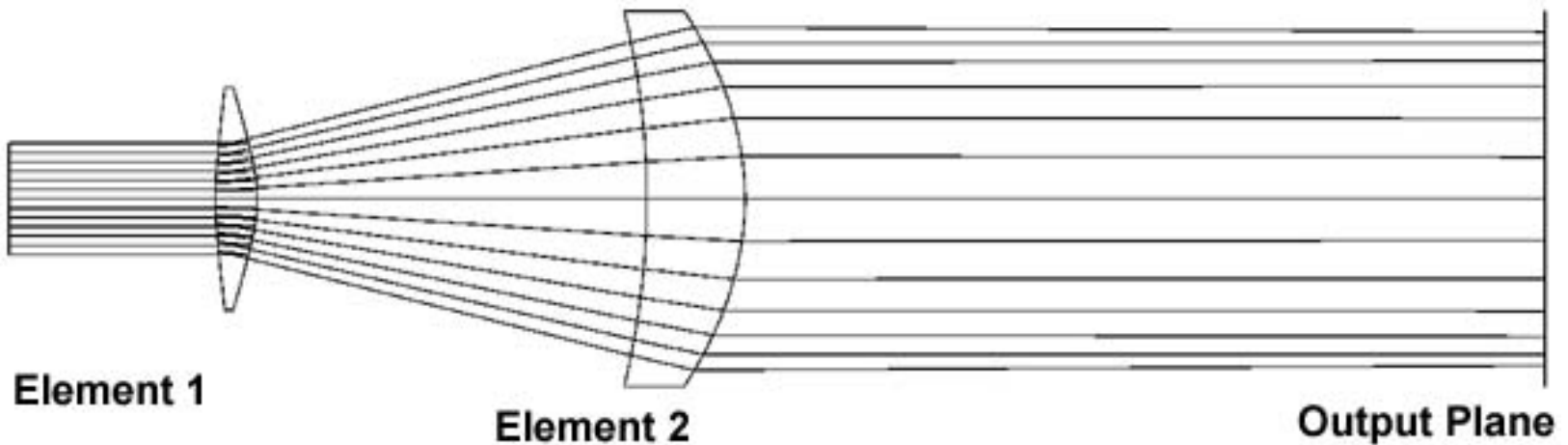

Figure 7. Cross-section of optical layout for the designed 2-element GRADIUM GRIN laser beam shaper where surface contours have non-zero conic constants

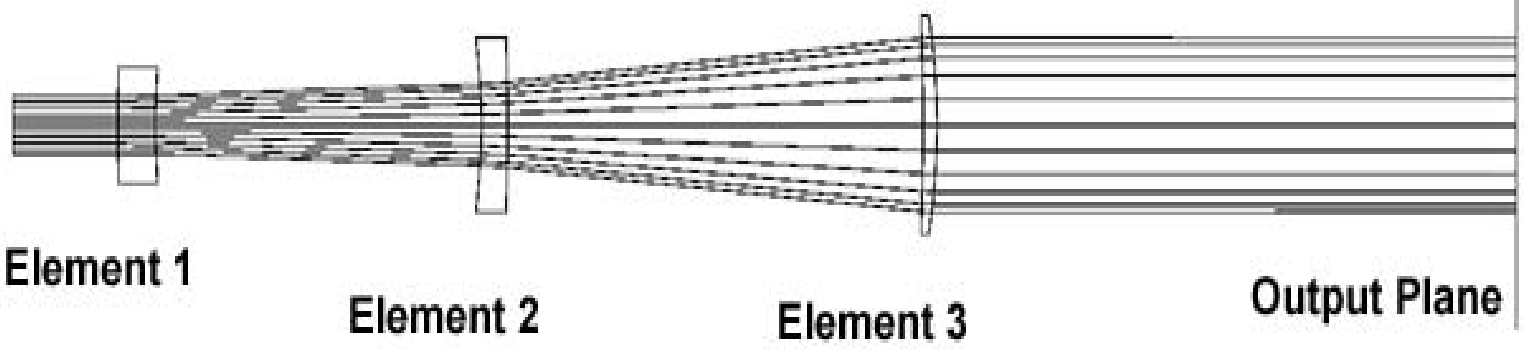

Figure 8. Cross sectional optical layout of the designed 3-element GRADIUM GRIN laser beam shaper with non-zero conic constants for each surface

Copyright 2004 Society of Photo-Optical Instrumentation Engineers.

This paper will be published in Proc. SPIE 5525, Sept. 2006, and is made available as an electronic preprint with permission of SPIE. One print or electronic copy may be made for personal use only. Systematic or multiple reproduction, distribution to multiple locations via electronic or other means, duplication of any material in the paper for a fee or for commercial purposes, or modification of the content of the paper are prohibited. 
Table 2. The designs of the 2- and 3-elements cataloged GRADIUM GRIN lens laser beam shapers.

\begin{tabular}{|c|c|c|c|}
\hline & 2-element GRIN lens & 3-element GRIN lens \\
\hline First lens & G14SFP & G23SFN & 217.6977 \\
\hline Radius of curvature of the first surface & -9.731856 & 1347.557 \\
Conic constant of the first surface & -10.87177 & 0.5 \\
Delta z of the first surface & 3.1387648 & 34.48442 \\
Radius of curvature of the second surface & 22.10039 & -120.7448 \\
Conic constant of the second surface & -73.89429 & G32SFN & -48.30423 \\
\hline Second lens & G41SFN & -43.11117 \\
\hline Radius of curvature of the first surface & -56.21458 & 1.3 & -124.1126 \\
Conic constant of the first surface & -0.4647533 & -1804 \\
Delta z of the first surface & 1.9871265 & $\mathrm{G} 14 \mathrm{SFP}$ \\
Radius of curvature of the second surface & -20.83304 & -199.5166 \\
Conic constant of the second surface & -0.935074 & -667.4521 \\
\hline Third lens & & 0.9 & -70.07896 \\
\hline Radius of curvature of the first surface & & -11.26095 \\
Conic constant of the first surface & & 6.443626 \\
Delta z of the first surface & & 4.514702 \\
Radius of curvature of the second surface & & 2.464079 \\
Conic constant of the second surface & & 56.4627 \\
\hline Thickness of first lens & -3.041133 & 71.79415 \\
Thickness of second lens & 7.124515 & & \\
Thickness of third lens & & & \\
Separation of 1st and 2nd lens & & & \\
Separation of 2nd and 3rd lens & & & \\
\hline
\end{tabular}

\section{CONCLUSION}

The conservation of energy within a bundle of rays has been applied to obtain a theoretical intercept with the output plane for a given incident ray position. This theoretical ray intercept with the output plane along with the constant optical path length condition of geometric optics have been used to define a beam shaping merit function. This beam shaping merit function has been used to design several configurations of 2- and 3-element GRIN laser beam shapers, which convert a single-mode Gaussian input beam into a flat-top output beam. The ZEMAX $^{22}$ global and hammer optimization programs have been used to minimize the laser beam shaping merit function for a two plano-aspheric beam shaper with known solutions and for several 2- and 3-element GRIN laser beam shapers with satisfactory performance as analyzed by ZEMAX optical design and analysis software. These examples have shown that the proposed optimization method is an effective way to design laser beam shaping systems when geometrical optics applies.

\section{REFERENCES}

1. P. W. Malyak, "Two-mirror unobscured optical system for reshaping the irradiance distribution of a laser beam," Appl. Opt. 31, pp. 4377-4383, 1992.

2. K. Nemoto, T. Fujii, N. Goto, H. Takino, T. Kobayashi, N. Shibata, K. Yamamura, and Y. Mori, "Laser beam intensity profile transformation with a fabricated mirror," Appl. Opt. 36, pp. 551-557, 1997.

3. D. L. Shealy and S. H. Chao, "Design and analysis of an elliptical gaussian laser beam shaping system," in Laser Beam Shaping II, F. M. Dickey and D. L. Shealy, eds., Proc. SPIE 4443, pp. 24-35, 2001.

4. D. L. Shealy and S. H. Chao, "Geometric optics-based design of laser beam shapers," Opt. Eng. 42, pp. 3123$3138,2003$.

Copyright 2004 Society of Photo-Optical Instrumentation Engineers.

This paper will be published in Proc. SPIE 5525, Sept. 2006, and is made available as an electronic preprint with permission of SPIE. One print or electronic copy may be made for personal use only. Systematic or multiple reproduction, distribution to multiple locations via electronic or other means, duplication of any material in the paper for a fee or for commercial purposes, or modification of the content of the paper are prohibited. 
5. B. R. Frieden, "Lossless conversion of a plane laser wave to a plane wave of uniform irradiance," Appl. Opt. 4, pp. 1400-1403, 1965.

6. P. W. Rhodes and D. L. Shealy, "Design and analysis of refractive optical systems for irradiance redistribution of collimated radiation," Appl.Opt. 19, pp. 3545-3553, 1980.

7. D. Shafer, "Gaussian to flat-top intensity distributing lens," Opt. Laser Technol. 14, pp. 159-160, 1982.

8. W. Jiang, D. L. Shealy, and J. C. Martin, "Design and testing of a refractive reshaping system," in Current Developments in Optical Design and Optical Engineering III, R. E. Fischer and W. J. Smith, eds., Proc. SPIE 2000, pp. 64-75, 1993.

9. C. Wang and D. L. Shealy, "Design of gradient-index lens systems for laser beam reshaping," Appl. Opt. 32, pp. 4763-4769, 1993.

10. J. A. Haffnagle and C. M. Jefferson, "Design and performance of a refractive optical system that converts a gaussian to a flattop beam," Appl. Opt. 39.30, pp. 5488-5499, 2000.

11. N. C. Evans and D. L. Shealy, "Design of a gradient-index beam shaping system via a genetic algorithm optimization method," in Laser Beam Shaping, F. M. Dickey and S. A. Holswade, eds., Proc. SPIE 4095, pp. 26-39, 2000.

12. W. B. Veldkamp, "Laser beam profile shaping with interlaced binary diffraction gratings," Appl. Opt. 21, pp. 3209-3212, 1982.

13. C.Y. Han, Y. Ishii, and K. Murata, "Reshaping collimated laser beams with gaussian profile to uniform profiles," Appl. Opt. 22, pp. 3644-3647, 1983.

14. C. C. Aleksoff, K. K. Ellis, and B. D. Neagle, "Holographic conversion of a gaussian beam to a near-field uniform beam," Opt. Eng. 30, pp. 537-543, 1991.

15. J. Cordingley, "Application of a binary diffractive optic for beam shaping on semiconductor processing by lasers," Appl. Opt. 32, pp. 2538-2542, 1993.

16. X. Tan, B. Gu, G. Yang, and B. Dong, "Diffractive phase elements for beam shaping: A new design method," Appl. Opt. 34, pp. 1314-1320, 1995.

17. N. C. Evans and D. L. Shealy, "Design and optimization of an irradiance profile-shaping system with a genetic algorithm method," Appl. Opt. 37, pp. 5216-5221, 1998.

18. D. E. Goldberg, Genetic Algorithms in Search, Optimization, and Machine Learning, Addison-Wesley, Reading, MA, 1989.

19. E. Betensky, "Postmodern lens design," Opt. Eng. 32, pp. 1750-1756, 1993.

20. K. Nemoto, T. Nayuki, T. Fujii, N. Goto, and Y. Kanai, "Optinum control of the laser beam intensity profile with a deformable mirror," Appl. Opt. 36, pp. 7689-7695, 1997.

21. F. M. Dickey and S. C. Holswade, Laser Beam Shaping: Theory and Techniques, Marcel Dekker, Inc., New York, 2000.

22. ZEMAX is a registered trademark of ZEMAX Development Corporation, 4901 Morena Blvd., Suite 207, San Diego, CA, 92117-7320 USA. See http://www.zemax.com for more information.

Copyright 2004 Society of Photo-Optical Instrumentation Engineers.

This paper will be published in Proc. SPIE 5525, Sept. 2006, and is made available as an electronic preprint with permission of SPIE. One print or electronic copy may be made for personal use only. Systematic or multiple reproduction, distribution to multiple locations via electronic or other means, duplication of any material in the paper for a fee or for commercial purposes, or modification of the content of the paper are prohibited. 